\title{
DISPERSANTES QUÍMICOS NA ANÁLISE GRANULOMÉTRICA DE LATOSSOLOS ${ }^{(1)}$
}

\author{
Jocimar Mauri (2), Hugo Alberto Ruiz ${ }^{(3)}$, Raphael \\ Bragança Alves Fernandes ${ }^{(4)}$, João Carlos Ker ${ }^{(3)}$ \& Luiz \\ Rogério Martins Rezende ${ }^{(5)}$
}

\begin{abstract}
RESUMO
A completa dispersão da amostra e a manutenção da estabilidade da fase dispersa são condições fundamentais para obtenção de maior acurácia na análise textural de amostras de solo. Para isso, foram testados compostos de $\mathrm{Na}^{+}$e $\mathrm{Li}^{+}$ como dispersantes químicos alternativos, frente à solução de $\mathrm{NaOH} 10 \mathrm{mmol} \mathrm{L}^{-1}$, com o objetivo de lograr dispersão mais efetiva e minimizar a presença de pseudocomponentes, fundamentalmente pseudossilte, na análise granulométrica de Latossolos. O trabalho foi realizado utilizando amostras de oito Latossolos do Estado de Minas Gerais, selecionados por apresentarem diferentes materiais de origem e teores de óxidos de ferro. Os tratamentos foram dispostos segundo um delineamento em blocos casualizados, correspondendo a um arranjo fatorial $8 \times 2 \times 7$, com três repetições. Os fatores em estudo foram as amostras dos oito Latossolos, retiradas nos horizontes A e B, e sete dispersantes. $\mathrm{O} \mathrm{NaOH}$ foi usado como dispersante de referência, testando-se alternativamente soluções de $\mathrm{LiOH}$, $\mathrm{NaClO},[\mathrm{NaClO}+\mathrm{NaOH}],[\mathrm{HCl}+\mathrm{NaOH}],\left(\mathrm{NaPO}_{3}\right)_{\mathrm{n}}$ e $\left[\left(\mathrm{NaPO}_{3}\right)_{\mathrm{n}}+\mathrm{NaOH}\right]$. Utilizando a maior proporção de argila para identificar a efetividade dos dispersantes, nenhum deles igualou-se ao $\mathrm{NaOH}$. Empregando o sinal $>$ para indicar maior efetividade, a sequência determinada foi: $\mathrm{NaOH}>\left[\left(\mathrm{NaPO}_{3}\right)_{\mathrm{n}}+\mathrm{NaOH}\right]>[\mathrm{HCl}+\mathrm{NaOH}]>[\mathrm{NaClO}+$ $\mathrm{NaOH}]>\mathrm{LiOH}>\left(\mathrm{NaPO}_{3}\right)_{\mathrm{n}}>\mathrm{NaClO}$. Dentre os dispersantes alternativos, destacouse a solução de $\left[\left(\mathrm{NaPO}_{3}\right)_{n}+\mathrm{NaOH}\right]$, com resultados próximos aos do dispersante de referência, evidenciando a importância da elevação do $\mathrm{pH}$ até valores próximos de 12. A efetividade das outras soluções sódicas com $\mathrm{pH}$ elevado, $[\mathrm{NaClO}+\mathrm{NaOH}]$ e $[\mathrm{HCl}+\mathrm{NaOH}]$, foi reduzida pela elevação da pressão osmótica da solução,
\end{abstract}

\footnotetext{
(1) Parte da Dissertação de Mestrado do primeiro autor apresentada ao Programa de Pós-Graduação em Solos e Nutrição de Plantas da Universidade Federal de Viçosa - UFV. Apoio financeiro FAPEMIG e CNPq. Recebido para publicação em 29 de abril de 2010 e aprovado em 16 de maio de 2011.

(2) Engenheiro Agrônomo/RTV Syngenta Brasil. Av. Ana Pena de Faria 427, CEP 35300-103 Caratinga (MG). E-mail: jocimar.mauri@syngenta.com

(3) Professor do Departamento de Solos, Universidade Federal de Viçosa - UFV. Peter Henry Rolfs s/n, CEP 36570-000 Viçosa (MG). Bolsista do CNPq. E-mails: hruiz@ufv.br; jcker@ufv.br

(4) Professor do Departamento de Solos, UFV. E-mail: raphael@ufv.br

${ }^{(5)}$ Discente de Agronomia, UFV. E-mail: luizmr@yahoo.com.br
} 
dificultando a dispersão da amostra de solo. $\mathrm{O}$ LiOH foi inferior ao dispersante de referência. Essa resposta é justificada pela tendência desse cátion alcalino de formar ligações covalentes que bloqueiam cargas elétricas negativas do complexo de troca das argilas, com diminuição da densidade de carga superficial. $\mathrm{O}\left(\mathrm{NaPO}_{3}\right)_{\mathrm{n}}$ e $\mathrm{NaClO}$ foram os dispersantes menos efetivos, pelo menor $\mathrm{pH}$ das suas soluções. Conclui-se que a solução de $\mathrm{NaOH}$ é a mais efetiva para dispersar amostras de solos com argilas que apresentam capacidade de troca catiônica dependente do pH, por favorecer o incremento de cargas negativas, a repulsão entre partículas e, consequentemente, a dispersão da amostra de solo, com valores relativamente baixos da pressão osmótica da suspensão.

Termos de indexação: análise textural, hidróxido de sódio, hidróxido de lítio, hexametafosfato de sódio, hipoclorito de sódio, ácido clorídrico.

\section{SUMMARY: CHEMICAL DISPERSION IN PARTICLE SIZE ANALYSIS OF LATOSOLS}

The complete sample dispersion and maintenance of stability in the dispersed phase are fundamental for a greater accuracy in soil texture analysis. For this purpose, $\mathrm{Na}^{+}$and $\mathrm{Li}^{+}$ compounds were tested as alternative chemical dispersants, compared to a $10 \mathrm{mmol} \mathrm{L}^{-1} \mathrm{NaOH}$ solution, for the possibility of a more effective dispersion and to minimize the presence of pseudo components, basically pseudo-silt, in the particle size analysis of Oxisols. Eight samples of Oxisols from the State of Minas Gerais, selected for their different parent materials and iron oxide contents were used. The treatments were arranged in a randomized block design, corresponding to a $2 \times 8 \times 7$ factorial arrangement, with three replications. The factors consisted of eight Oxisol samples, collected in the horizons $A$ and $B$, and of seven dispersants. NaOH was used as reference dispersant and the alternative dispersants tested were $\mathrm{LiOH}, \mathrm{NaClO}$, [NaClO $+\mathrm{NaOH}],[\mathrm{HCl}+\mathrm{NaOH}],(\mathrm{NaPO})_{n}$ and $\left[(\mathrm{NaPO})_{3}+\mathrm{NaOH}\right]$. None of these identified the effectiveness of dispersants as well as $\mathrm{NaOH}$ when using the highest clay proportion. The sequence of effectiveness was ranked as follows: $\mathrm{NaOH}>\left[\left(\mathrm{NaPO}_{3}\right)_{n}+\mathrm{NaOH}\right]>[\mathrm{HCl}+\mathrm{NaOH}]$ $>[\mathrm{NaClO}+\mathrm{NaOH}]>\mathrm{LiOH}>\left(\mathrm{NaPO}_{3}\right)_{n}>\mathrm{NaClO}$. Among the alternative dispersants, the solution $\left[\left(\mathrm{NaPO}_{3}\right)_{n}+\mathrm{NaOH}\right]$ performed similarly to the reference dispersant, highlighting the importance of raising the $\mathrm{pH}$ values to near 12. The effectiveness of the other sodic solutions with high $\mathrm{pH},[\mathrm{NaClO}+\mathrm{NaOH}]$ and $[\mathrm{HCl}+\mathrm{NaOH}]$, was reduced when raising the osmotic pressure of the solution, hampering soil dispersion. The performance of LiOH was worse than of the reference. This response can be explained by the tendency of this alkali cation to form covalent bonds that block negative electric charges from the clay exchange complex, decreasing surface charge density. The dispersants $\left(\mathrm{NaPO}_{3}\right)_{n}$ and $\mathrm{NaClO}$ were the least effective, due to the lower solution $\mathrm{pH}$. It was concluded that $\mathrm{NaOH}$ is most effective in dispersing soil with clays with $\mathrm{pH}$-related cation exchange capacity, since it tends to increase negative charges, repulsion between particles and consequently soil sample dispersion, at a relatively low osmotic pressure of the suspension.

Index terms: Soil texture analysis, sodium hydroxide, lithium hydroxide, sodium hexametaphosphate, sodium hypochlorite, hydrochloric acid.

\section{INTRODUÇÃO}

A textura representa as proporções das frações areia, silte e argila do solo. Sua determinação, realizada em laboratório, envolve pré-tratamentos, dispersão da amostra e separação e quantificação das frações texturais. O conhecimento da textura é importante, por apresentar aplicações no uso e manejo dos solos, na melhor utilização da água em sistemas produtivos, nos estudos de gênese e classificação de solos, nas práticas de fertilização e correção do solo, no desenvolvimento e rendimento das culturas agrícolas e na avaliação da dinâmica de nutrientes e poluentes no solo.

Os pré-tratamentos têm por objetivo remover os agentes cimentantes, orgânicos (substâncias húmicas) e inorgânicos (óxidos de ferro e de alumínio mal cristalizados e carbonatos, principalmente). Aplicase também pré-tratamento para a remoção de agentes floculantes, como no caso da análise granulométrica 
de solos afetados por sais, uma vez que a elevada concentração salina na solução do solo reduz a dupla camada difusa e favorece a floculação. Entretanto, os pré-tratamentos, ainda que contribuam para a quebra dos pequenos agregados de solo, não são normalmente utilizados na análise de rotina, iniciando-se a análise textural com a dispersão da amostra. Depois disso, a separação das frações texturais é realizada por peneiramento, no caso da areia, e por sedimentação, para o silte e a argila.

A dispersão é o processo de separação das partículas reunidas em agregados, em partículas individuais (areia, silte e argila). Essa fase da análise textural envolve a combinação de processos físicos e químicos. A dispersão física ou mecânica consiste na agitação da suspensão de solo, o que contribui para a quebra dos pequenos agregados.

No que diz respeito à dispersão química, o $\mathrm{pH}$ do meio é de grande importância na dispersão de argilas que apresentam cargas dependentes de $\mathrm{pH}$. Nesse caso, a dispersão será facilitada por valores de $\mathrm{pH}$ elevados e superiores ao ponto de carga zero (PCZ) do solo. Nessas condições, eleva-se a proporção de cargas negativas dependentes do $\mathrm{pH}$ e, consequentemente, incrementa-se a repulsão entre as partículas.

A presença de óxidos de ferro e alumínio, os quais apresentam PCZ elevados, sendo os primeiros entre 7 e 9 (Schwertmann \& Taylor, 1989) e os últimos entre 8 e 9,2 (Hsu, 1989), leva o PCZ médio do solo a valores próximos de seu $\mathrm{pH}$, o que dificulta a dispersão das partículas (Raij \& Peech, 1972). Esse fato é de importância particular para os solos de regiões tropicais úmidas, principalmente para os Latossolos, que apresentam argilas com carga variável e, portanto, são dependentes de $\mathrm{pH}$. Além disso, por causa da elevada proporção de $\mathrm{Al}$ trocável, nesses solos o $\mathrm{NaOH}$ é o dispersante mais utilizado (Freire, 1963; Vettori \& Pierantoni, 1968). A elevação do $\mathrm{pH}$ incrementa as cargas negativas dependentes do $\mathrm{pH}$ e, também, precipita o $\mathrm{Al}^{3+}$ como $\mathrm{Al}(\mathrm{OH})_{3}$, diminuindo a pressão osmótica da solução, com consequente melhora na dispersão.

Procurando conseguir suspensões estáveis, elevase o potencial zeta pela substituição dos cátions do complexo de troca por um cátion dispersante, monovalente e de elevado raio iônico hidratado (Kirkham \& Powers, 1972). Esse procedimento leva à expansão da dupla camada difusa. Assim, quando o complexo de troca estiver saturado com $\mathrm{Na}^{+}$, haverá condição favorável à dispersão. Contudo, concentrações elevadas de $\mathrm{Na}^{+}$na solução de equilíbrio levam à diminuição do potencial osmótico, podendo promover a compressão da dupla camada difusa e, consequentemente, a floculação.

Para obter uma suspensão de solo efetivamente dispersa, há necessidade de substituir os cátions floculantes, como $\mathrm{Al}^{3+}, \mathrm{Ca}^{2+} \mathrm{e} \mathrm{Mg}^{2+}$, frequentemente encontrados saturando as argilas, por cátions monovalentes de maior raio iônico hidratado. $\mathrm{Na}$ dispersão, a sequência $\mathrm{Li}^{+}>\mathrm{Na}^{+}>\mathrm{K}^{+}>\mathrm{Rb}^{+}>\mathrm{Cs}^{+}$ indica a ordem decrescente esperada de eficiência para amostras de solos (Baver et al., 1972).

$\mathrm{Na}$ dispersão química, os agentes dispersantes mais utilizados são os compostos de $\mathrm{Na}$, como o hexametafosfato e o hidróxido de sódio. A escolha do dispersante dependerá dos cátions presentes no complexo de troca e da relação entre cargas permanentes e cargas dependentes do $\mathrm{pH}$ nos minerais da fração argila. Em solos com elevados teores de $\mathrm{Ca}^{2+}{\mathrm{e} \mathrm{Mg}^{2+}}^{2+}$ e,ou, predominância de cargas permanentes que apresentam dificuldade na estabilização da suspensão, o hexametafosfato de sódio é o dispersante mais indicado. Esse sal fornece o $\mathrm{Na}^{+}$necessário à dispersão e também diminui a pressão osmótica da solução, por precipitação dos fosfatos ligados aos cátions alcalinoterrosos (Baver et al., 1972).

Como indicado, o $\mathrm{NaOH}$ é o dispersante mais utilizado na análise textural de solos característicos de regiões tropicais e úmidas (Freire, 1963; Vettori \& Pierantoni, 1968). Nesses solos, o incremento de cargas negativas com a elevação e manutenção do $\mathrm{pH}$ em valores elevados é fundamental para estabilizar a suspensão.

Procurando identificar a concentração de $\mathrm{NaOH}$ mais adequada para a dispersão de amostras de solos, Jucksch et al. (1995) testaram concentrações na faixa de 3 a $100 \mathrm{mmol} \mathrm{L}^{-1}$, utilizando agitação rápida, a 12.000 rotações por minuto, durante $15 \mathrm{~min}$; em amostras de quatro classes de solos, sendo dois gibbsíticos - Latossolo Vermelho distrófico e Latossolo Vermelho-Amarelo distrófico - e dois cauliníticos - Latossolo Amarelo e Argissolo Amarelo, classes designadas de acordo com o atual Sistema Brasileiro de Classificação de Solos (Embrapa, 2006). Esses autores concluíram que, para os solos estudados, a concentração de $10 \mathrm{mmol} \mathrm{L}^{-1}$ foi a mais efetiva na dispersão da fração argila e na estabilidade da suspensão, o que confirma a eficiência dessa concentração indicada para análises de rotina (Vettori, 1969).

$\mathrm{O} \mathrm{LiOH}$, pelo maior raio iônico hidratado do $\mathrm{Li}^{+}$ em relação ao $\mathrm{Na}^{+}$, possivelmente apresentaria vantagens sobre o $\mathrm{NaOH}$ na dispersão de Latossolos. Entretanto, Donagemma et al. (1998) não obtiveram sucesso ao testarem essa base em agregados de um Latossolo Vermelho-Escuro, em concentração equivalente à do $\mathrm{NaOH}$, habitualmente utilizada na rotina (Vettori, 1969). Esses autores observaram que nas amostras avaliadas o $\mathrm{LiOH}$ foi menos eficiente que o $\mathrm{NaOH}$ na dispersão de argila, quando comparados na mesma concentração. Assim, concluíram haver necessidade de novos estudos com esse dispersante, visando estabelecer condições mais adequadas para sua utilização na análise textural.

Diante desse contexto, o presente estudo apresenta como hipóteses:

- O pH elevado da solução de referência $(\mathrm{NaOH}$ $10 \mathrm{mmol} \mathrm{L}^{-1}$ ) é determinante na dispersão de solos 
com elevada capacidade de troca catiônica dependente de $\mathrm{pH}$.

- Considerando o mesmo pH:

- $\mathrm{OLi}^{+}$é dispersante químico mais efetivo que o $\mathrm{Na}^{+}$, devido a seu maior raio iônico hidratado;

- O NaClO é dispersante químico mais efetivo que o $\mathrm{NaOH}$, por oxidar parcialmente as substâncias orgânicas cimentantes; $\mathrm{e}$

- O tratamento prévio com $\mathrm{HCl}$ permite dispersão mais efetiva, por dissolver parcialmente os óxidos de $\mathrm{Fe}$ e $\mathrm{Al}$ mal cristalizados.

- As afirmativas previamente indicadas são condicionadas pela pressão osmótica da solução final, visto que seu incremento pode favorecer a floculação de argilas.

O objetivo deste trabalho foi testar compostos de $\mathrm{Na}^{+}$e Li ${ }^{+}$como dispersantes químicos alternativos, frente à solução de $\mathrm{NaOH} 10 \mathrm{mmol} \mathrm{L}^{-1}$, buscando alcançar dispersão mais efetiva que minimize a presença de pseudocomponentes, fundamentalmente pseudossilte, na análise granulométrica de Latossolos.

\section{MATERIAL E MÉTODOS}

Foram utilizadas amostras dos horizontes A e B de oito Latossolos argilosos e muito argilosos do Estado de Minas Gerais, selecionados por apresentarem diferentes materiais de origem (Quadro 1). As amostras foram coletadas, secas ao ar e passadas em peneira de $2 \mathrm{~mm}$. Os tratamentos foram dispostos segundo um delineamento em blocos casualizados, correspondendo a um arranjo fatorial $8 \times 2 \times 7$, com três repetições. Os fatores em estudo foram as amostras dos oito Latossolos indicados (Quadro 1), retiradas nos horizontes A e B, e sete dispersantes (Quadro 2).

$\mathrm{O} \mathrm{NaOH} 10 \mathrm{mmol} \mathrm{L}^{-1}$, normalmente utilizado na análise granulométrica (Vettori, 1969), foi considerado dispersante de referência. A composição dos dispersantes alternativos foi ajustada de forma a igualar a concentração ou o pH da solução de referência (Quadro 2). Esse ajuste foi realizado numa etapa preliminar, determinando-se as concentrações de $\mathrm{Na}$ ou de $\mathrm{Li}$, por fotometria de emissão de chama, e o $\mathrm{pH}$, por potenciometria. Em acréscimo, determinou-se a pressão osmótica das sete soluções em estudo, por microcrioscopia.

As análises granulométricas foram realizadas segundo a rotina do Laboratório de Física do Solo da Universidade Federal de Viçosa, conforme descrito em Donagemma et al. (2003), utilizando-se o peneiramento para a fração areia e a sedimentação para as frações argila e silte. Resumidamente, $50 \mathrm{~mL}$ de solução de cada dispersante avaliado foram adicionados a $10 \mathrm{~g}$ de terra fina seca. Nas amostras de solo que receberam $\mathrm{HCl}$ e $\mathrm{NaOH}$, a incorporação foi sequencial, sendo previamente incorporados $10 \mathrm{~mL}$ de $\mathrm{HCl}$ 0,25 $\mathrm{mol} \mathrm{L}^{-1}$ e $15 \mathrm{~mL}$ de água deionizada, atuando como prétratamento para remoção de óxidos de ferro e alumínio mal cristalizados; após $8 \mathrm{~h}$, foram adicionados $25 \mathrm{~mL}$ de $\mathrm{NaOH} 0,3 \mathrm{~mol} \mathrm{~L}^{-1}$, visando à dispersão. A dispersão mecânica que se seguiu foi promovida via agitação lenta em agitador rotatório tipo Wagner, durante $16 \mathrm{~h}$ e a $50 \mathrm{rpm}$. A fração silte foi obtida por diferença entre duas pipetagens, sendo na primeira recolhido silte + argila e, na segunda, apenas argila, considerando o tempo de sedimentação segundo a equação de Stokes, levando-se em conta a densidade de partículas (Embrapa, 1997) de cada solo em estudo. As frações texturais foram calculadas conforme proposta apresentada por Ruiz (2005).

Para avaliação dos resultados foi realizada análise de variância, considerando conjuntamente os dados obtidos para os oito solos e os dois horizontes estudados. A opção pela análise conjunta de solos e horizontes deveu-se à intenção de se buscar avaliar o efeito dos dispersantes testados com maior número de amostras. Considera-se que um laboratório de rotina de análises físicas não tem conhecimento prévio da natureza das amostras que recebe, portanto, não é capaz de identificar com segurança o horizonte e a classe de

Quadro 1. Identificação dos oito Latossolos de Minas Gerais

\begin{tabular}{|c|c|c|c|c|c|c|}
\hline \multirow{2}{*}{ Codificação } & \multirow{2}{*}{ Classe de solo } & \multirow{2}{*}{ Material de origem } & \multirow{2}{*}{ Localização } & \multicolumn{3}{|c|}{ Composição textural $^{(1)}$} \\
\hline & & & & Areia & Silte & Argila \\
\hline & & & & \multicolumn{3}{|c|}{$\mathrm{kg} \mathrm{kg}^{-1}$} \\
\hline LVj1 & Latossolo Vermelho perférrico & Itabirito & Nova Lima & 0,293 & 0,177 & 0,530 \\
\hline $\mathrm{LVj} 2$ & Latossolo Vermelho perférrico & Tufito e Conglomerados Cineríticos & Patos de Minas & 0,143 & 0,320 & 0,537 \\
\hline $\mathrm{LVw}$ & Latossolo Vermelho acriférrico & Tufito e Conglomerados Cineríticos & Patos de Minas & 0,136 & 0,156 & 0,708 \\
\hline LVdf1 & Latossolo Vermelho distroférrico & Anfibolito & Abre Campo & 0,166 & 0,140 & 0,694 \\
\hline LVdf2 & Latossolo Vermelho distroférrico & Gabro & Lavras & 0,167 & 0,071 & 0,762 \\
\hline LVAdf1 & Latossolo Vermelho-Amarelo distroférrico & Gnaisse & Viçosa & 0,198 & 0,017 & 0,785 \\
\hline LVAdf2 & Latossolo Vermelho-Amarelo distroférrico & Cobertura argilosa sobre tufito & São Gotardo & 0,079 & 0,109 & 0,812 \\
\hline LVA & Latossolo Vermelho-Amarelo & Calcário & Paraopeba & 0,035 & 0,405 & 0,560 \\
\hline
\end{tabular}

(1) Determinação textural em amostras do horizonte B dos solos estudados, utilizando-se $\mathrm{NaOH}^{10} \mathrm{mmol} \mathrm{L}^{-1}$ como dispersante. Fonte: Donagemma et al. (2003). 
Quadro 2. Concentração e pH das soluções dos dispersantes químicos alternativos ajustados aos valores da solução de referência $(\mathrm{NaOH})$ e pressão osmótica das soluções resultantes ${ }^{(1)}$

\begin{tabular}{lcrc}
\hline Dispersante & Concentração & $\mathbf{p H}$ & Pressão osmótica \\
\hline & $\mathrm{mmol} \mathrm{L}$ & & $\mathrm{kPa}$ \\
$\mathrm{NaOH}$ & 10 & 11,8 & 45 \\
$\mathrm{LiOH}$ & 10 & 11,8 & 44 \\
$\mathrm{NaClO}$ & 10 & 8,6 & 48 \\
$\mathrm{NaClO}+\mathrm{NaOH}$ & $10+10,9$ & 11,8 & 97 \\
$\mathrm{HCl}+\mathrm{NaOH}^{-1}$ & $5+15$ & 11,8 & 63 \\
$(\mathrm{NaPO})_{\mathrm{n}}$ & 10 & 6,6 & 21 \\
$\left(\mathrm{NaPO}_{3}\right)_{\mathrm{n}}+\mathrm{NaOH}$ & $10+10$ & 11,8 & 56
\end{tabular}

(1) Concentração, $\mathrm{pH}$ e pressão osmótica referem-se à solução final com volume igual a $500 \mathrm{~mL} .{ }^{(2)}$ A solução de $\mathrm{NaOH}$ foi incorporada $8 \mathrm{~h}$ após adição da solução de $\mathrm{HCl}$.

solo a que elas pertencem. Nesse sentido, a avaliação geral de todos os dados torna-se relevante, de forma a permitir maior generalização das conclusões obtidas. O estudo mais detalhado dos efeitos obtidos em cada solo e horizonte é feito no trabalho de Mauri (2008). Na sequência da análise de variância, aplicou-se o teste de Dunnett para identificar diferenças estatisticamente significativas na comparação com o dispersante de referência ( $\left.\mathrm{NaOH} 10 \mathrm{mmol} \mathrm{L}^{-1}\right)$.

\section{RESULTADOS E DISCUSSÃO}

Os dispersantes alternativos testados resultaram na obtenção de proporções médias das frações granulométricas diferentes das obtidas com o tratamento de referência (Quadro 3), à exceção do uso da mistura $\left[\left(\mathrm{NaPO}_{3}\right)_{\mathrm{n}}+\mathrm{NaOH}\right]$, cujos resultados foram semelhantes aos obtidos com $\mathrm{NaOH}$.

A utilização de $\mathrm{NaClO}$ como dispersante alternativo (Quadro 2) respondeu à possibilidade deste de oxidar parcialmente a matéria orgânica do solo e, com isso, facilitar a dispersão da amostra, por redução no teor desse agente agregante. Essa oxidação tinha sido testada com resultados positivos por Donagemma (2000). Todavia, o método original (Anderson, 1963) exige aquecimento em banho-maria a $75^{\circ} \mathrm{C}$ durante período prolongado, filtração e secagem da amostra, antes de submetê-la à análise granulométrica.

$\mathrm{Na}$ tentativa de adequar o uso do $\mathrm{NaClO}$ a um método apropriado para realizar análises de rotina, com número elevado de amostras, Santos et al. (2001) experimentaram o $\mathrm{NaClO}$ em temperatura ambiente. $\mathrm{O}$ mesmo procedimento foi utilizado neste estudo, testando-se soluções de $\mathrm{NaClO}$ e de $\mathrm{NaClO}+\mathrm{NaOH}$. A presença do $\mathrm{NaClO}$ provocou oxidação parcial da matéria orgânica do solo (Quadro 4), porém a redução não foi muito acentuada, ficando na faixa de 0,3 a $22 \%$. Donagemma et al. (2003), utilizando o método original, registraram reduções da matéria orgânica entre 45 e 67 \%. Com respeito a Santos et al. (2001), que trabalharam com os mesmos solos de Donagemma et al. (2003), a redução teve valores variando de 11 e 47 \%. É importante destacar que Santos et al. (2001) utilizaram concentração de $\mathrm{NaClO}$ de $40 \mathrm{mmol} \mathrm{L}^{-1}$. A menor concentração empregada neste estudo, de $10 \mathrm{mmol} \mathrm{L}-1$, justifica a menor oxidação da matéria orgânica, diante dos resultados de Santos et al. (2001).

A utilização de $\mathrm{HCl}$ e, na sequência, após 8 h, $\mathrm{NaOH}$ como dispersante respondeu à possibilidade de dissolver parcialmente óxidos de ferro e alumínio mal cristalizados, reduzindo a proporção desses cimentos nas amostras de solos. Essa dissolução parcial tinha sido testada por Donagemma et al. (2003), com resultados promissores para a análise granulométrica. Contudo, na forma indicada na proposta original, a análise não era adequada para utilização em rotina de laboratório, com grande número de amostras. Tanto no trabalho de Donagemma et al. (2003) como nesta pesquisa, a redução do teor de óxidos de ferro após aplicação desse pré-tratamento foi mínima (Quadro 4).

Quadro 3. Teores médios de areia grossa, areia fina, silte e argila dos oito solos e dois horizontes (n=48), determinados com os sete dispersantes químicos estudados

\begin{tabular}{|c|c|c|c|c|}
\hline Dispersante & Areia grossa & Areia fina & Silte & Argila \\
\hline & \multicolumn{4}{|c|}{$-\mathrm{kg} \mathrm{kg}^{-1}$} \\
\hline $\mathrm{NaOH}$ & 0,103 & 0,072 & 0,184 & 0,641 \\
\hline$\left(\mathrm{NaPO}_{3}\right)_{\mathrm{n}}+\mathrm{NaOH}$ & 0,106 & 0,071 & 0,186 & 0,638 \\
\hline $\mathrm{NaClO}+\mathrm{NaOH}$ & 0,104 & 0,070 & $0,198^{*}$ & $0,628^{*}$ \\
\hline $\mathrm{HCl}+\mathrm{NaOH}$ & 0,105 & 0,070 & $0,198^{*}$ & 0,627 * \\
\hline $\mathrm{LiOH}$ & $0,110^{*}$ & $0,081^{*}$ & $0,203^{*}$ & $0,606^{*}$ \\
\hline$\left(\mathrm{NaPO}_{3}\right)_{\mathrm{n}}$ & $0,118^{*}$ & $0,083^{*}$ & $0,204^{*}$ & $0,595^{*}$ \\
\hline $\mathrm{NaClO}$ & $0,134^{*}$ & $0,099^{*}$ & $0,358^{*}$ & $0,409^{*}$ \\
\hline $\mathrm{dms}^{(1)}$ & 0,004 & 0,004 & 0,006 & 0,008 \\
\hline
\end{tabular}

(1) Diferença mínima significativa na comparação com o dispersante de referência $(\mathrm{NaOH})$. * Diferenças estatisticamente significativas a $5 \%$ pelo teste de Dunnett na comparação dos dispersantes alternativos com a solução de $\mathrm{NaOH}$. 
Quadro 4. Teores de carbono orgânico total e ferro extraído por solução de oxalato de amônio, determinados em amostras que receberam $\mathrm{NaClO}$ e $\mathrm{HCl}$, respectivamente

\begin{tabular}{|c|c|c|c|c|c|c|}
\hline \multirow{2}{*}{ Solo } & \multirow{2}{*}{ Horizonte } & \multicolumn{3}{|c|}{ Carbono orgânico total } & \multicolumn{2}{|c|}{ Fe Oxalato } \\
\hline & & Testemunha $^{(1)}$ & $\mathrm{NaClO}$ & $\mathrm{NaClO}+\mathrm{NaOH}$ & Testemunha & $\mathrm{HCl}+\mathrm{NaOH}$ \\
\hline & & & & $\mathrm{g} \mathrm{kg}^{-1}$ & & \\
\hline LVj1 & $\begin{array}{l}\mathrm{A} \\
\mathrm{B}\end{array}$ & $\begin{array}{r}24,4 \\
8,2\end{array}$ & $\begin{array}{r}21,6 \\
7,4\end{array}$ & $\begin{array}{r}24,2 \\
7,5\end{array}$ & $\begin{array}{l}1,7 \\
1,7\end{array}$ & $\begin{array}{l}1,5 \\
1,5\end{array}$ \\
\hline $\mathrm{LVj} 2$ & $\begin{array}{l}\text { A } \\
\text { B }\end{array}$ & $\begin{array}{r}20,3 \\
4,8\end{array}$ & $\begin{array}{r}19,5 \\
4,0\end{array}$ & $\begin{array}{r}18,6 \\
4,4\end{array}$ & $\begin{array}{l}1,9 \\
1,6\end{array}$ & $\begin{array}{l}1,5 \\
1,5\end{array}$ \\
\hline $\mathrm{LVw}$ & $\begin{array}{l}\text { A } \\
\text { B }\end{array}$ & $\begin{array}{l}20,9 \\
13,4\end{array}$ & $\begin{array}{l}18,9 \\
13,1\end{array}$ & $\begin{array}{l}19,9 \\
12,8\end{array}$ & $\begin{array}{l}1,1 \\
1,1\end{array}$ & $\begin{array}{l}0,9 \\
0,9\end{array}$ \\
\hline LVdf1 & $\begin{array}{l}\text { A } \\
\text { B }\end{array}$ & $\begin{array}{l}40,7 \\
16,4\end{array}$ & $\begin{array}{l}38,2 \\
14,7\end{array}$ & $\begin{array}{l}36,3 \\
15,4\end{array}$ & $\begin{array}{l}1,0 \\
0,7\end{array}$ & $\begin{array}{l}1,0 \\
0,6\end{array}$ \\
\hline LVdf2 & $\begin{array}{l}\text { A } \\
\text { B }\end{array}$ & $\begin{array}{r}24,7 \\
5,8\end{array}$ & $\begin{array}{r}24,2 \\
5,2\end{array}$ & $\begin{array}{r}23,0 \\
4,5\end{array}$ & $\begin{array}{l}0,7 \\
0,4\end{array}$ & $\begin{array}{l}0,7 \\
0,2\end{array}$ \\
\hline LVAdf1 & $\begin{array}{l}\text { A } \\
\text { B }\end{array}$ & $\begin{array}{r}25,5 \\
6,7\end{array}$ & $\begin{array}{r}25,4 \\
6,1\end{array}$ & $\begin{array}{r}24,4 \\
6,3\end{array}$ & $\begin{array}{l}0,9 \\
0,2\end{array}$ & $\begin{array}{l}0,7 \\
0,1\end{array}$ \\
\hline LVAdf2 & $\begin{array}{l}\text { A } \\
\text { B }\end{array}$ & $\begin{array}{l}25,5 \\
10,6\end{array}$ & $\begin{array}{l}24,9 \\
10,5\end{array}$ & $\begin{array}{l}24,5 \\
10,2\end{array}$ & $\begin{array}{l}0,6 \\
0,3\end{array}$ & $\begin{array}{l}0,5 \\
0,2\end{array}$ \\
\hline LVA & $\begin{array}{l}\text { A } \\
\text { B }\end{array}$ & $\begin{array}{l}35,0 \\
13,5\end{array}$ & $\begin{array}{l}34,9 \\
12,9\end{array}$ & $\begin{array}{l}29,4 \\
12,8\end{array}$ & $\begin{array}{l}0,8 \\
0,7\end{array}$ & $\begin{array}{l}0,6 \\
0,5\end{array}$ \\
\hline
\end{tabular}

(1) Determinações em amostras de $10 \mathrm{~g}$ de TFSA agitada com $50 \mathrm{~mL}$ de água deionizada por $16 \mathrm{~h}$ a $50 \mathrm{rpm}$.

Como indicado por Donagemma et al. (2003), o resultado mais acurado da análise textural depende da completa dispersão da amostra de solo e da manutenção da estabilidade da fase dispersa. Assim, considerando os Latossolos em análise, o incremento na proporção de argilas é considerado indicativo de maior efetividade do tratamento imposto, pela diminuição na proporção de pseudocomponentes, fundamentalmente pseudossilte.

Considerando os oito solos e dois horizontes, foi observado que os seis dispersantes alternativos possivelmente sofreram interferência da matéria orgânica, pois foram obtidas proporções inferiores de argila em 24 oportunidades, no horizonte A, e em 15, no horizonte B (Figura 1). Isso foi acompanhado por incrementos nas proporções de areia grossa, areia fina e silte nos horizontes A: 13, 12 e 16, respectivamente; no horizonte $B$, foram 5,7 e 14 , respectivamente (Mauri, 2008). Deve ressalvar-se que o total de incrementos - 41 para o horizonte A e 26 para o horizonte $\mathrm{B}$ - não é igual às reduções no teor de argilas (Figura 1), pois essa diminuição é acompanhada, eventualmente, de mais de um incremento das outras frações texturais. Os 16 incrementos líquidos indicados para o silte no horizonte A resultaram de 20 valores superiores e 4 inferiores, e os 14 do horizonte B, de 15 e 1, respectivamente (Mauri, 2008).

Tomando como referência a proporção de argila, observa-se que nenhum dispersante alternativo apresentou efetividade superior à solução de $\mathrm{NaOH}-$ no máximo, respostas semelhantes (Quadro 3). Empregando o sinal > para indicar maior efetividade na comparação com o dispersante de referência, a sequência de eficiência obtida foi a seguinte:

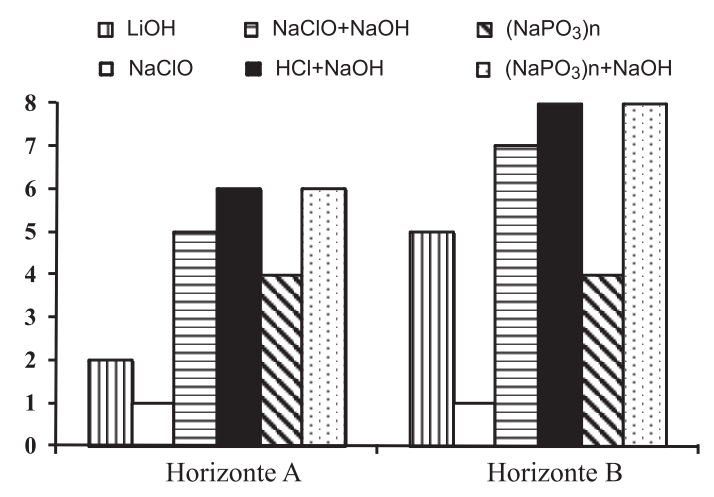

Figura 1. Número de amostras de solo em que os dispersantes alternativos apresentaram resposta igual à do dispersante de referência $(\mathrm{NaOH})$ na determinação do teor de argila (avaliações com dispersantes alternativos: total $=96$, sendo 48 por horizonte e 8 por solo).

$$
\begin{aligned}
& \mathrm{NaOH} \geq\left[\left(\mathrm{NaPO}_{3}\right)_{\mathrm{n}}+\mathrm{NaOH}\right]>[\mathrm{HCl}+\mathrm{NaOH}]> \\
& {[\mathrm{NaClO}+\mathrm{NaOH}]>\mathrm{LiOH}>\left(\mathrm{NaPO}_{3}\right)_{\mathrm{n}}>\mathrm{NaClO}}
\end{aligned}
$$

Os dados apresentados justificam a importância da elevação do pH na dispersão de amostras de solo com elevada proporção de capacidade de troca catiônica dependente do $\mathrm{pH}$. Os dispersantes alternativos mais destacados foram o que igualaram o pH da solução de referência e não a concentração de sódio (Quadro 2). $\mathrm{O} \mathrm{LiOH}$ teve resposta abaixo do esperado (Figura 1).

Pelos dados apresentados na figura 1 e quadro 3 , destaca-se a solução de $\left[\left(\mathrm{NaPO}_{3}\right)_{\mathrm{n}}+\mathrm{NaOH}\right]$, com menor pressão osmótica entre os compostos sódicos 
com pH 11,8 (Quadro 2), como eventual dispersante que possa permitir utilização mais difundida em todas as regiões do Brasil. É de importância a pesquisa com solos da região Nordeste, com algumas amostras que apresentam predomínio de $\mathrm{Ca}^{2+}$ no complexo de troca, comparando a dispersão com $\mathrm{NaOH},\left[\left(\mathrm{NaPO}_{3}\right)_{\mathrm{n}}\right.$ $\left.+\mathrm{Na}_{2} \mathrm{CO}_{3}\right]$ e $\left[\left(\mathrm{NaPO}_{3}\right)_{\mathrm{n}}+\mathrm{NaOH}\right]$.

Os dispersantes $(\mathrm{NaClO}+\mathrm{NaOH})$ e $(\mathrm{HCl}+\mathrm{NaOH})$ com $\mathrm{pH}$ elevado, ajustado àquele do dispersante de referência (Quadro 2), não mostraram suficiente efetividade na dispersão (Quadro 3 e Figura 1), razão pela qual podem ser descartados como alternativas úteis. Suas soluções apresentaram as maiores pressões osmóticas entre todas as testadas (Quadro 2).

Análise diferenciada exige o LiOH (Quadro 3 e Figura 1). $\mathrm{O} \mathrm{Li}^{+}$é o primeiro elemento dos metais alcalinos (grupo 1A da tabela periódica), com raio iônico hidratado maior que o do $\mathrm{Na}^{+}$. Sob esse aspecto, o $\mathrm{Li}^{+}$seria dispersante mais efetivo que o $\mathrm{Na}^{+}$. Contudo, sob alguns aspectos, a química do $\mathrm{Li}^{+}$ aproxima-se da do $\mathrm{Mg}^{2+}$ (Cotton \& Wilkinson, 1966); dessa forma, o $\mathrm{Li}^{+}$seria menos efetivo que o $\mathrm{Na}^{+}$na dispersão. Segundo Cotton \& Wilkinson (1966), as particularidades do $\mathrm{Li}^{+}$, com respeito aos outros metais alcalinos, resultam principalmente do pequeno tamanho do íon, que, entre outras características, leva à singular e apreciável tendência à formação de ligações covalentes. Essas ligações bloqueiam cargas elétricas negativas do complexo de troca das argilas e, por ser estáveis, reduzem apreciavelmente a repulsão entre as partículas de argila, pela diminuição da densidade de carga superficial.

A baixa eficiência do $\left(\mathrm{NaPO}_{3}\right)_{\mathrm{n}}$ e do $\mathrm{NaClO}$, sem elevação do $\mathrm{pH}$ (Quadro 3 e Figura 1), mostra a indiscutível necessidade de se trabalhar com soluções sódicas de forte alcalinidade na dispersão de amostras de solo com capacidade de troca catiônica com elevada proporção de cargas dependentes do $\mathrm{pH}$.

\section{CONCLUSÕES}

1. $\mathrm{O} \mathrm{NaOH} 10 \mathrm{mmol} \mathrm{L}^{-1}$, dispersante de referência, foi o mais efetivo - comprovado pela maior proporção de argilas nas análises texturais dos horizontes A e B das amostras dos oito Latossolos estudados.

2. A mistura $\left[\left(\mathrm{NaPO}_{3}\right)_{\mathrm{n}}+\mathrm{NaOH}\right]$ dispersou adequadamente as amostras dos Latossolos, com resposta semelhante ao $\mathrm{NaOH}$. Poderia, assim, ser utilizada e deveria ser testada, por apresentar características promissoras para utilização em outras classes de solo, como os normalmente encontrados na região Nordeste.

3. As outras misturas $[\mathrm{NaClO}+\mathrm{NaOH}]$ e $[\mathrm{HCl}+$ $\mathrm{NaOH}$, mesmo apresentando pH elevado, não se mostraram tão efetivas quanto o $\mathrm{NaOH}$. Sua efetividade foi reduzida pela elevação da pressão osmótica da solução, não sendo, portanto, recomendada para esses solos.
4. Os dispersantes sem o $\mathrm{NaOH}\left(\mathrm{LiOH},\left(\mathrm{NaPO}_{3}\right)_{\mathrm{n}}\right.$ e $\mathrm{NaClO}$ ) foram os menos efetivos na dispersão dos Latossolos: o Li, por formar ligações covalentes que bloqueiam cargas elétricas negativas do complexo de troca, e os dois restantes, por apresentarem baixo $\mathrm{pH}$.

\section{LITERATURA CITADA}

ANDERSON, J.U. An improved pretreatment for mineralogical analysis of samples containing organic matter. Clays Clay Miner., 10:380-388, 1963.

BAVER, L.D., GARDNER, W.H. \& GARDNER, W.R. Soil physics. 4.ed. New York, John Wiley, 1972. 498p.

COTTON, F.A. \& WILKINSON, G. Advanced inorganic chemistry, a comprehensive text. New York, Interscience, 1966. $1136 \mathrm{p}$.

DONAGEMMA, G.K. Pré-tratamento na análise textural visando a minimização do pseudo-silte em Latossolos de Minas Gerais. Viçosa, MG, Universidade Federal de Viçosa, 2000. 89p. (Tese de Mestrado)

DONAGEMMA, G.K.,; RUIZ, H.A.; KER, J.C. \& COELHO, F.C. Utilização do hidróxido de lítio como dispersante na análise textural de diferentes classes de agregados de um Latossolo Vermelho-Escuro. In: REUNIÃO BRASILEIRA DE MANEJO E CONSERVAÇÃO DO SOLO E DA ÁGUA, 12., 1998, Fortaleza, CE. Resumos expandidos. Fortaleza, Sociedade Brasileira de Ciência do Solo, 1998. p.36-37.

DONAGEMMA, G.K.; RUIZ, H.A.; FONTES, M.P.F.; KER, J.C. \& SCHAEFER, C.E.G.R. Dispersão de Latossolos em resposta à utilização de pré-tratamentos na análise textural. R. Bras. Ci. Solo, 27:765-772, 2003.

EMPRESA BRASILEIRA DE PESQUISA AGROPECUÁRIA EMBRAPA. Manual de métodos de análise de solo. 2.ed. Rio de Janeiro, Centro Nacional de Pesquisa de Solos, 1997. 212p.

EMPRESA BRASILEIRA DE PESQUISA AGROPECUÁRIA . EMBRAPA. Sistema brasileiro de classificação de solos. 2.ed. Brasília, Embrapa-SPI; Rio de Janeiro, Centro Nacional de Pesquisa de Solos, 2006. 306p.

FREIRE, O. Dispersão de solos, estudo comparativo de tratamentos químicos empregados no preparo de amostras para análise mecânica. Piracicaba, Universidade de São Paulo/Escola Superior de Agricultura Luiz de Queiroz, 1963. 168p. (Tese de Doutorado)

HSU, P.H. Aluminum hydroxides and oxyhydroxides. In: DIXON, J.B. \& WEED, S.B., eds. Minerals in soil environments. Madison, Soil Science Society of America, 1989. v.7. p.331-378.

JUCKSCH, I.; COSTA, L.M.; MELLO, J.W.V.; BUENO, B.S. \& FONTES, L.E.F. Meios mecânicos e concentração de $\mathrm{NaOH}$ na dispersão e estabilidade de suspensões de argila. In: CONGRESSO BRASILEIRO DE CIÊNCIA DO SOLO, 25., Viçosa, MG, 1995. Resumos expandidos. Viçosa, MG, Sociedade Brasileira de Ciência do Solo, 1995. v.1. p.104105. 
KIRKHAM, D. \& POWERS, W.L. Advanced soil physics. 2.ed. New York, Interscience, 1972. 548p.

MAURI, J. Dispersantes químicos na análise granulométrica de Latossolos. Viçosa, MG, Universidade Federal de Viçosa, 2008. 46p. (Tese de Mestrado)

RAIJ, B.van \& PEECH, M. Electrochemical properties of some Oxisols and Alfisols of tropics. Soil Sci. Soc. Am. Proc., 36:587-598, 1972.

RUIZ, H.A. Incremento da exatidão da análise granulométrica do solo por meio da coleta da suspensão (silte + argila). R. Bras. Ci. Solo, 29:297-300, 2005.

SANTOS, B.C.M.; RUIZ, H.A. \& DONAGEMMA, G.K. Uso do hipoclorito de sódio como dispersante na análise textural de Latossolos de Minas Gerais. In: CONGRESSO BRASILEIRO DE CIÊNCIA DO SOLO, 28., Londrina, 2001. Resumos. Londrina, Sociedade Brasileira de Ciência do Solo, 2001. p.15.
SCHWERTMANN, U. \& TAYLOR, R.M. Iron oxides. In: DIXON, J.B. \& WEED, S.B., eds. Minerals in soil environments. 2.ed. Madison, Soil Science Society of America, 1989. p.379-438.

VETTORI, L. Métodos de análise de solo. Rio de Janeiro, Ministério da Agricultura, 1969. 24p. (Boletim Técnico, 9)

VETTORI, L. \& PIERANTONI, H. Análise granulométrica: novo método para determinar a fração argila. Rio de Janeiro, Equipe de Pedologia e Fertilidade do Solo, Ministério da Agricultura, 1968. 9p. (Boletim Técnico, 3) 